\title{
FORMULATION AND EVALUATION OF FLOATING-MUCOADHESIVE MICROSPHERES OF NOVEL NATURAL POLYSACCHARIDE FOR SITE SPECIFIC DELIVERY OF RANITIDINE HYDROCHLORIDE
}

\author{
VIKRAM KUMAR SAHU ${ }^{1}$, NITIN SHARMA ${ }^{2}$, PRATAP KUMAR SAHU ${ }^{3}$, SHUBHINI A. SARAF ${ }^{4}$
}

1Department of Pharmacy, Maharana Pratap College of Pharmacy, Kanpur, 209217, India, ${ }^{2}$ Meerut Institute of Engineering and Technology, NH-58, Baghpat Crossing, Partapur Bypass Road, Meerut, 250005, India, ${ }^{3}$ School of Pharmaceutical Sciences, Siksha 'O' Anusandhan University, Bhubaneswar, 751030, India, ${ }^{4}$ Department of Pharmaceutical Sciences, Babasaheb Bhimrao Ambedkar University, Lucknow, 226025, India

Email: vikramsahuknp@gmail.com

Received: 12 Nov 2016, Revised and Accepted: 17 Apr 2017

ABSTRACT

Objective: Localization of ranitidine hydrochloride (RH) into the upper part of the intestinal tract is beneficial for better drug bioavailability. Present work described the method of preparation of novel plant polysaccharide based floating microspheres for delivery of the drug into the stomach.

Methods: Polysaccharide was extracted from the seeds of plant Tamarindus indica (TI). Extracted polysaccharide was evaluated for some physicochemical parameters. Floating-mucoadhesive microspheres were prepared by using extracted polysaccharide as mucoadhesive excipients while eudragit as a release controlling polymers by using emulsion crosslinking method. Chemical crosslinking was done by using epichlorohydrin. Prepared microspheres were evaluated for their drug-polymer compatibility study by using fourier transform infrared spectroscopy (FT-IR). Further characterization such as size, surface properties, swelling index, percentage encapsulation, in vitro buoyancy and drug release was performed.

Results: FT-IR study confirms the chemical crosslinking of extracted polysaccharide and also drug stability during processing of microspheres. The size of microspheres was in the range of 5.38 to $7.84 \mu \mathrm{m}$. SEM images revealed that all batches were of spherical in size and smooth surface. The swelling index showed better swelling in the range of 158-257 percentages. Encapsulation efficiency was found to be decreased by decreasing the concentration of polysaccharide. In vitro buoyancy study possesses that formulation F1 showed better floating ability as compared to the others. Finally, in vitro drug release study revealed that prepared microspheres were able to release the $100 \%$ drug within $8-12$ h, indicating sustain release behavior.

Conclusion: Present study concludes that polysaccharide of TI may be used as excipients for the preparation of floating-mucoadhesive microspheres.

Keywords: Tamarindus indica, Ranitidine hydrochloride, Floating microspheres, Natural polymer

(c) 2017 The Authors. Published by Innovare Academic Sciences Pvt Ltd. This is an open access article under the CC BY license (http://creativecommons.org/licenses/by/4.0/) DOI: http://dx.doi.org/10.22159/ijap.2017v9i3.16137

\section{INTRODUCTION}

$\mathrm{RH}$ is a commonly used antacid as $\mathrm{H}_{2}$ receptor antagonist which shows maximum absorption at the initial part of small intestine. Moreover, colonic metabolism is also responsible for poor oral drug bioavailability [1]. Effective treatment can be achieved by administration of $200 \mathrm{mg}$ of $\mathrm{RH}$, three times a day, which leads to a patient in compliance. In order to improve therapeutic action and patient compliance, it is required to design a formulation which can increase the retention of the drug into the upper part of along with sustain release action up to $12 \mathrm{~h}$ [2].

The floating system is used for localized drug release into the specific region of the gastrointestinal tract (GIT). This system can be effervescent or non-effervescent depends on ingredients used in the delivery system. The most commonly used excipients in noneffervescent floating drug delivery system are either swellable or gel forming hydrocolloids and polysaccharide. These systems are also known as plug type system due to the tendency to remain lodged near the pyrrolic sphincter [3]. Nowadays, a large number of plant polysaccharide has been investigated for their potential role in the floating drug delivery system. These polymers have a tendency to swell on gastric media and float, therefore, increasing the residential time of formulation into the upper part of GIT. Additionally, this plant polysaccharide has some advantages such as biodegradable, biocompatible, low cost and relatively free availability [4].

With the same context, in the present work drug loaded floating mucoadhesive microspheres were developed to improve the residential time of RH into upper GIT. A novel plant polysaccharide was investigated for their application in the mucoadhesive delivery system. The polysaccharide was isolated from the seeds of TI. The polysaccharide of TI is nonionic, neutral, branched structure consist of cellulose like backbone which carries xylose and galactoxylose subunits. Polysaccharide has been investigated for many applications such as gelling agent, tablet binder, stabilizer and thickening agent $[5,6]$. So far, the polysaccharide is not evaluated in the floating drug delivery system. Aim of present work was to investigate the role of novel TI polysaccharide in development of floating mucoadhesive microspheres for localized drug delivery of RH specifically into upper parts of GIT.

\section{MATERIALS AND METHODS}

RH was obtained as generous gift sample from Hetero labs, Hyderabad, India. Seeds of TI were purchased from local market of Kanpur (UP). All other materials such as Eudragit, castor oil, epicholorhydrin, and sodium bicarbonate were of analytical grade and were procured from commercial sources.

\section{Polysaccharide extraction}

TI polysaccharide was extracted as per reported methods in various publications [7]. The seed were process by separating the brown peels from the kernel seed with the blender and plastic sieve were used to separate the seed. The seed were powdered and boiled with distilled water. Mucilaginous juice was separated by filtration through muslin cloth under vacuum and precipitated by ethanol. Precipitate was dried at $50{ }^{\circ} \mathrm{C}$ for $2 \mathrm{~d}$. Further, the extracted dry polysaccharide was characterised in terms of purity and viscosity. 
The purity of the polysaccharide was evaluated by testing the presence of carbohydrate, by performing the molisch's, ruthenium, and iodine test. The viscosity of $2 \% \mathrm{w} / \mathrm{v}$ aqueous solution of polysaccharide was determined by using rheometer (Brookfield R/S plus, Massachusetts, USA) of cone and plate geometry equipped with C-25 din measuring spindle at a shear rate $10\left(\mathrm{~s}^{-1}\right)$ for $100 \mathrm{sec}$ at $34 \pm 0.5^{\circ} \mathrm{C}$ temperature.

\section{Preparation of microspheres}

Microspheres were prepared by using cross-linking emulsification method described by Nitin et al. [8]. The accurately weighed amount of $\mathrm{RH}$ was dissolved into distilled water to make $20 \mathrm{mg} / \mathrm{ml}$ drug solution.
Varied amount of extracted polysaccharide and eudragit were dispersed into drug solution to make $2 \% \mathrm{w} / \mathrm{v}$ dispersion of both excipients (TI polysaccharide and eudragit) (table 1). Sodium bicarbonate (0.1:1with TI polysaccharide) was dissolved into aqueous phase.

Aqueous phase was allowed for complete hydration till $4 \mathrm{~h}$ under stirring using a magnetic stirrer. Hydrated aqueous phase was emulsified in castor oil (1:10 aqueous to oil phase ratio) using span 80 $(1.0 \% \mathrm{v} / \mathrm{v})$ at high-speed homogenizer (2000 rpm, Yamato, LT400, Tokyo, Japan). Sulphuric acid $(0.2 \mathrm{ml}$ ) and epichlorohydrin (as chemical crosslinker, $4.0 \% \mathrm{v} / \mathrm{v}$ ) were added. And stirring was continued till $18 \mathrm{~h}$ at $45^{\circ} \mathrm{C}$. Finally, microspheres were collected by centrifugation followed by its washing using isopropyl alcohol and dried at $60^{\circ} \mathrm{C}$ for $5 \mathrm{~h}$.

Table 1: Batch detail and characterization of Tamarindus indica polysaccharide based floating-micrspheres

\begin{tabular}{llllll}
\hline $\begin{array}{l}\text { Formulation } \\
\text { code }\end{array}$ & $\begin{array}{l}\text { TI Polysaccharide } \\
(\mathbf{\% w} / \mathbf{w})\end{array}$ & $\begin{array}{l}\text { Eudragit } \\
(\mathbf{\%} / \mathbf{w})\end{array}$ & $\begin{array}{l}\text { Particle size } \\
(\boldsymbol{\mu m})\end{array}$ & $\begin{array}{l}\text { Swelling ratio } \\
(\%)\end{array}$ & $\begin{array}{l}\text { In vitro buoyancy } \\
(\mathbf{\%}) \mathbf{w})\end{array}$ \\
\hline F1 & 100 & - & $7.84 \pm 0.34$ & $257 \pm 26$ & $87.35 \pm 12.43$ \\
F2 & 75 & 25 & $3.51 \pm 0.11$ & $212 \pm 31$ & $81.72 \pm 18.29$ \\
F3 & 50 & 50 & $6.48 \pm 0.36$ & $187 \pm 13$ & $78.05 \pm 9.55$ \\
F4 & 25 & 75 & $5.38 \pm 0.52$ & $158 \pm 39$ & $76.40 \pm 5.49$ \\
\hline
\end{tabular}

*Percentage of TI polysaccharide and eudragit was for total content of both excipients to make $2 \% \mathrm{w} / \mathrm{v}$ dispersion into aqueous phase

\section{Characterization of microspheres}

\section{FT-IR study}

The FT-IR spectra of RH, extracted polysaccharide and prepared microspheres were recorded to determine any possible chemical changes of drug during microspheres preparation. Dried $\mathrm{KBr}$ was used as diluent and samples were scanned over the range of 4000 to 400 $\mathrm{cm}^{-1}$ by using FT-IR spectrophotometer (Shimadzu 84005, Japan).

\section{Morphology}

Surface morphology of prepared microspheres was studied using scanning electron microscopy (SEM). The samples were sprinkled on an aluminium stub by using double sided adhesive tape and then gold sputter coated at $50 \mathrm{~mA}$ under an argon atmosphere (POLARON model SC-76430). The thickness of the coating was $200 \AA$ Å. Images were captured in the magnification range of $3000 \mathrm{X}$ by using electron microscope (LEO-430, UK).

\section{Particle size analysis}

The particle size determination was done by using phase contrast microscope (Radical Instruments, Ambala Cant, India). Prepared microspheres were suspended into light paraffin oil. Few drops (23 ) of this suspension were shifted over the glass slide and covered with a coverslip. The diameter of approximate 300 microspheres was measured and the average diameter was calculated.

\section{Swelling ratio}

Swelling property of prepared floating microspheres was studied as per the method described by Rana et al. [9]. Briefly, known weight $\left(25 \mathrm{mg}\right.$ ) of microspheres were soaked at $37 \pm 0.5^{\circ} \mathrm{C}$ in phosphate buffer ( $\mathrm{pH}$ 1.2) solution. After a certain time, period microspheres were and excess media was removed by blotting with suitable media. Swollen microspheres were weighed by using electronic balance (Shimadzu AUX220, Japan). The degree of swelling ( $\alpha$ ) was calculated by using equation 1 .

$$
\alpha=\frac{\mathrm{Wg}-\mathrm{Wo}_{0}}{\mathrm{Wo}_{\mathrm{o}}} \times 100 \text { (1) }
$$

Where $W_{o}$ is the initial weight of microspheres and $W_{g}$ is the weight after swelling.

\section{In vitro buoyancy study}

In order to explore the floating ability of prepared microspheres in vitro buoyancy study was performed according to the method describe by Dey et al. [10]. The study was carried out in $0.1 \mathrm{~N} \mathrm{HCl}$ containing $0.02 \% \mathrm{v} / \mathrm{v}$ tween 80 . Prepared microspheres were dispersed into the $900 \mathrm{ml}$ of media at $37 \pm 0.5^{\circ} \mathrm{C}$. Paddle was rotated at 100 rotations per minute. Each fraction of microspheres floated on the surface and those settled down were recorded at a predetermined time interval. Settled microspheres were collected, dried and weighed.

\section{Encapsulation efficiency}

These parameters were estimated by dispersing accurately weighed quantity of crushed powder of prepared microspheres $(25 \mathrm{mg})$ into 25 $\mathrm{ml}$ distilled water. The dispersion was kept overnight with under continuous shaking for complete release of RH from microspheres. Debris of microspheres was removed by filtration and centrifugation. The absorbance of the filtrate was measured after suitable dilution using UV-visible spectrophotometer (Shimadzu 1700, Japan) at $313 \mathrm{~nm}$. Experiments were carried out in triplicate and the encapsulation efficiency and drug content were calculated by using equations 2 .

$$
\text { Encapsulation efficiency (\%) }=\frac{\text { actual amount of drug }}{\text { theoretical amount of drug }} \times 100(2
$$

\section{In vitro dissolution studies}

For determination of in vitro dissolution study, accurately weighed the quantity of prepared microspheres (equivalent to $300 \mathrm{mg}$ of $\mathrm{RH}$ ) was transferred in the beaker having $900 \mathrm{ml}$ phosphate buffer $(\mathrm{pH} 1.2)$, temperature $37 \pm 1{ }^{\circ} \mathrm{C}$; staring rate $100 \mathrm{rpm}$. At predetermined time intervals, aliquots of $1 \mathrm{ml}$ were withdrawn, replaced by equal volume of fresh media and centrifuged at $4000 \mathrm{~g}$ for $15 \mathrm{~min}$. Finally, the supernatant was estimated for drug content by using UV-visible spectrophotometer at $313 \mathrm{~nm}$. Further, the exact mechanism of drug release from the microspheres was calculated by fitting release data different release kinetic models such as zero-order, first-order, Higuchi's and Korsmeyer-Peppas equations. Data of release kinetics such as of kinetic rate constant $(\mathrm{k})$, correlation coefficient $\left(\mathrm{r}^{2}\right)$ and release exponent (n) were calculated for the determination of best fit model [11].

\section{RESULTS AND DISCUSSION}

The polysaccharide of TI was extracted by using precipitation method with percentage yield of $13.59 \% \mathrm{w} / \mathrm{w}$. The brownish red dry polysaccharide was characterized for their purity and viscosity. As a result, it was found that extracted polysaccharide was of carbohydrate in nature. Presence of mucilage and absence of any other ingredients indicates the high level of purity of extracted polysaccharide. Similarly, $2.0 \% \mathrm{w} / \mathrm{v}$ aqueous solution showed $304 \pm 21 \mathrm{mPas}$ viscosity at shear rate $10\left(\mathrm{~s}^{-1}\right)$ and $37 \pm 0.5{ }^{\circ} \mathrm{C}$ temperature. Junginger et al., (2007) suggested a relation between viscosity and mucoadhesion of polymers [12]. According to this relation a mucoadhesion property of polymer is only triggered by its swelling behavior. For effective mucoadhesion, polymer need to swell first, followed by interpenetration into biological tissue. Therefore, high value of viscosity indicates that the 
extracted polysaccharides may be considered as excipients for mucoadhesive drug delivery. Emulsification cross-linking method was found to be a suitable method for the preparation of RH loaded TI based microspheres. The percentage yield of prepared microspheres was in the range of $78-81 \% \mathrm{w} / \mathrm{w}$. Epichlorohydrin was used as a chemical cross linking agent, which has been used for cross-linking of various carbohydrates. Chemically, it is an organochlorine compound (an organic compound containing at least one covalent bonded atom of chlorine) and an epoxide (cyclic ether with three ring atom).

\section{FT-IR study}

The FT-IR spectra of RH, extracted polysaccharide and prepared microspheres are shown in fig. 1 . The characteristic peaks of RB and extracted polysaccharide were studied by using the previously reported literature [13]. Obtained FT-IR spectra of RH were compared with spectra of microspheres. The study indicates that all characteristic peaks of $\mathrm{RH}$ were present in the spectra of microspheres, which revealed that the drug was unchanged during the preparation of microspheres.

However, some new peaks were observed in the spectra of microspheres at frequencies 1164 and $867 \mathrm{~cm}^{-1}$, which may be due to the formation of mono and diether linkage as the result of chemical cross-linking between extracted polysaccharide and epichlorohydrin. The absence of any new peak in the FT-IR spectra of microspheres also confirmed the absence of any by-product through epichlorohydrin mediated polysaccharide cross linking.

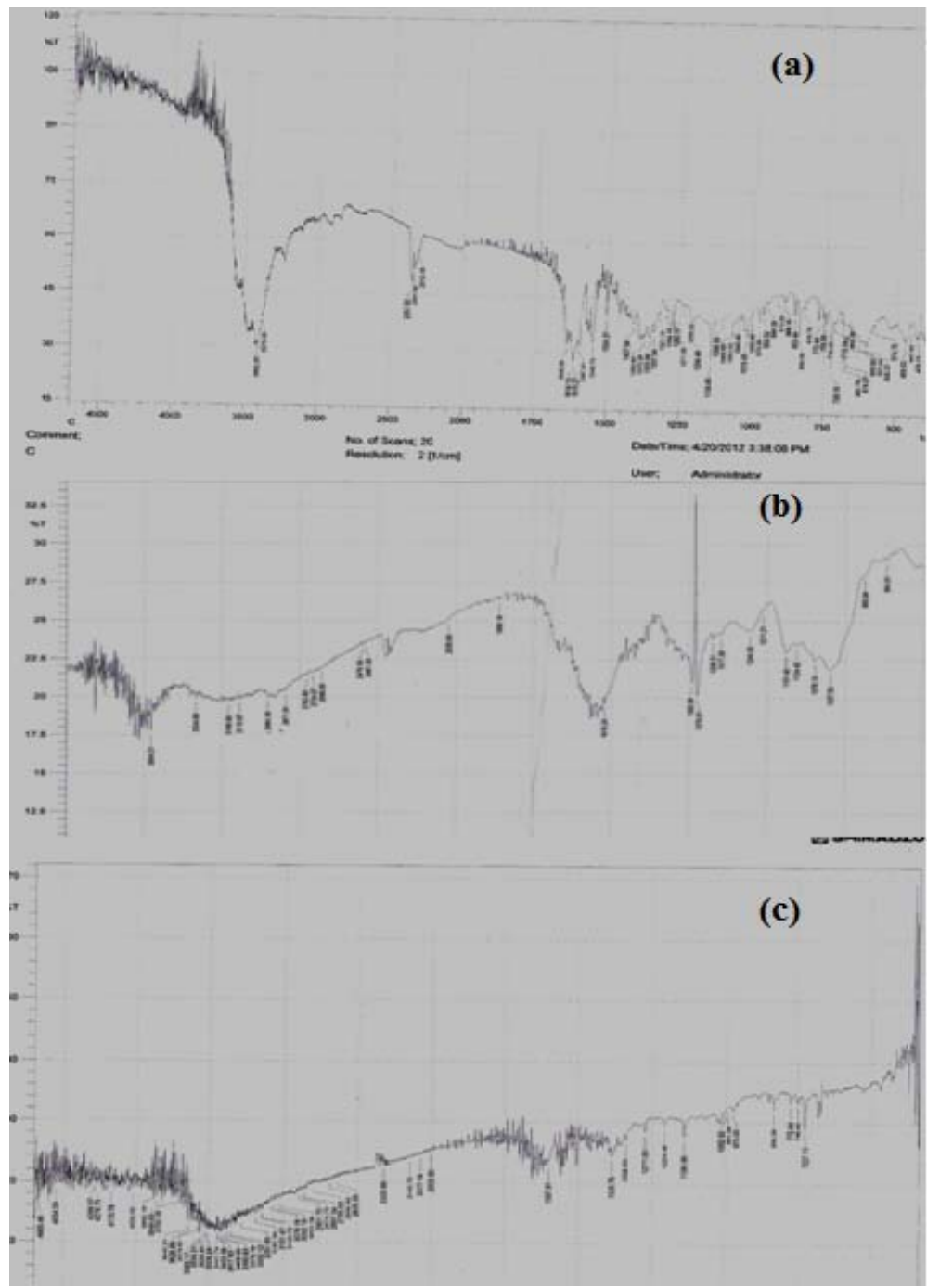

Fig. 1: FTIR spectra of drug RH (a), extracted plant polysaccharide (b) and prepared floating-mucoadhesive microspheres

\section{Surface morphology}

Surface characteristics of different batches of prepared TI polysaccharide based microspheres were examined by using scanning electron microscopy (SEM). Fig. 2 indicates the SEM images of prepared microspheres of all batches. Visual examination of the SEM pictures indicated that all batches of microspheres were of spherical shape with varied surface roughness. Present investigation indicates that there were no any significant effects of variables on surface morphology of microspheres.

\section{Particle size}

Particle sizes of all batches of drug loaded TI polysaccharide based microspheres were evaluated by using simple microscopic methods.
Particle size of all batches of microspheres was in the range of 5.38$7.84 \mu \mathrm{m}$ (table 1). The present study indicated that there was not any remarkable effect of concentration of excipients on the particle size of prepared microspheres.

\section{Swelling ratio}

Swelling behavior of microspheres defines the extent of mucoadhesion and drug release pattern through it. Therefore, evaluation of this study becomes essential while preparing any mucoadhesive formulations. The effect of varied concentration of TI polysaccharide and eudragit on the swelling index of the microspheres was evaluated (fig. 3). Fig. indicates that swelling index was gradually decreased by decreasing the concentration of TI 
polysaccharide. Batch F1 showed highest swelling, while batch F4 showed minimum swelling. This might be due to insufficient polysaccharide content to swell. Highest swelling index F1 may be due to highest polysaccharide concentration.
Since, polysaccharides are consist of large numbers of polar compound which leads to water absorption and holding capacity and further more swelling property of prepared microspheres [8].

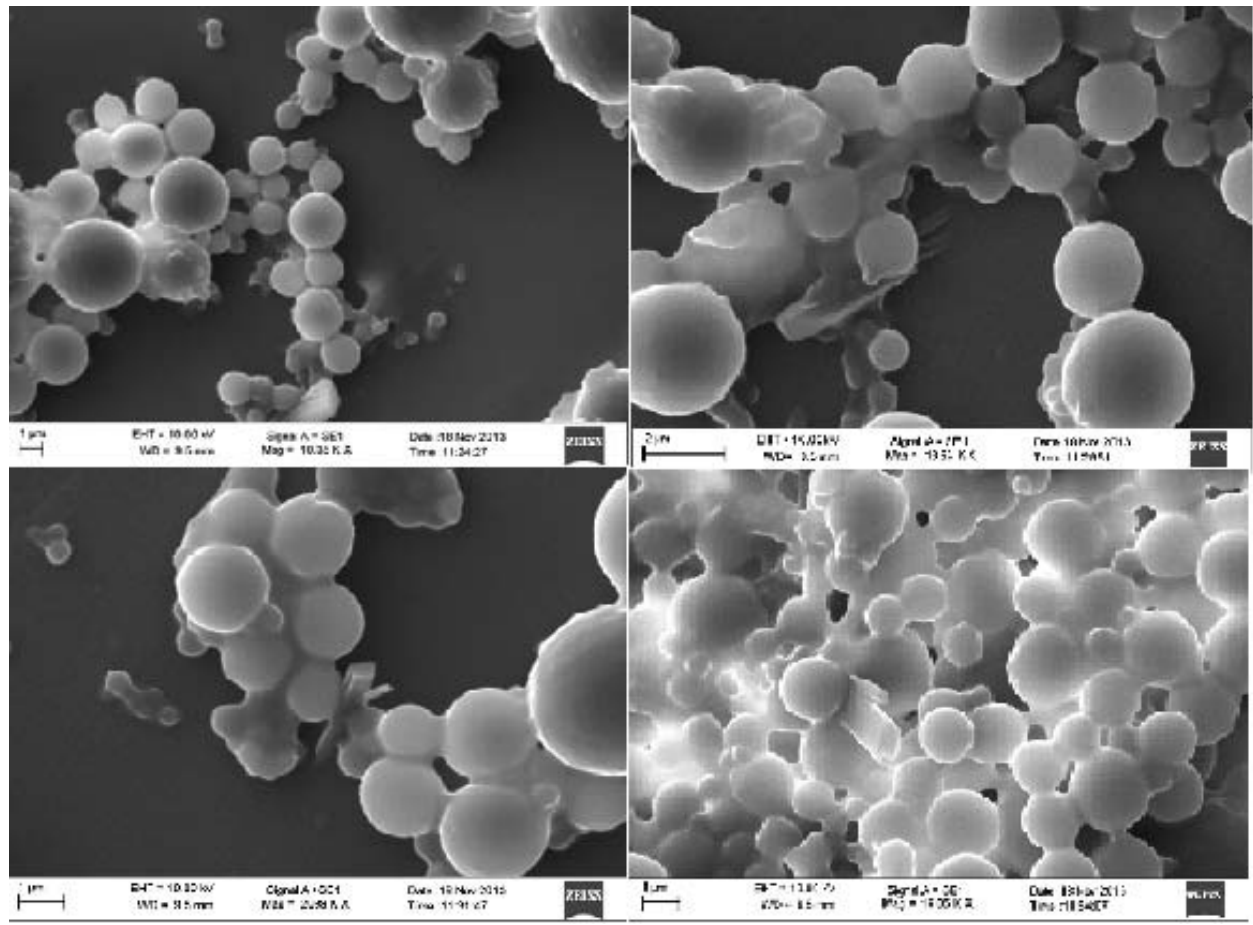

Fig. 2: SEM images of all batches of microspheres prepared by using extracted TI polysaccharide

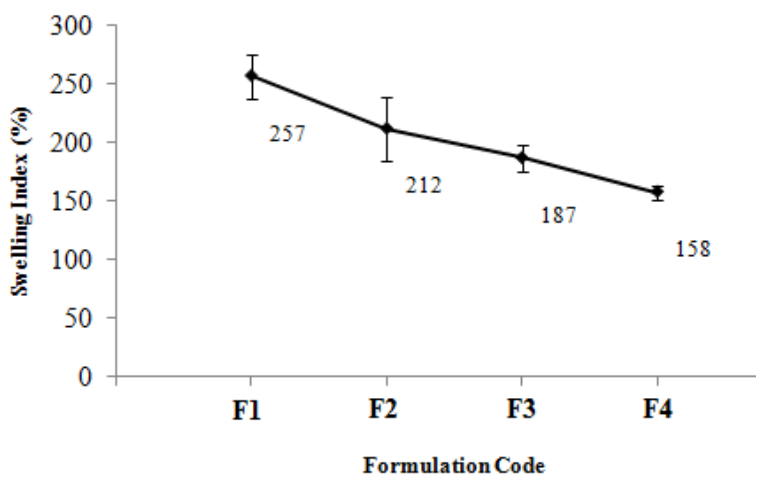

Fig. 3: Percentage swelling index of TI polysaccharide based floating-mucoadhesive microspheres measurement was done in triplicates $(n=3$, mean $\pm S D)$

\section{In vitro buoyancy study}

The purpose of preparing floating microspheres was to extend the gastric residence time of the drug. Floating ability of prepared microspheres was determined by using in vitro buoyancy study in $0.1 \mathrm{~N} \mathrm{HCl}$ containing $0.02 \% \mathrm{v} / \mathrm{v}$ tween 80 . Tween 80 was added to counteract the downward pulling at the liquid surface by lowering surface tension. The prepared microspheres floated immediately on the medium, and the floating lag time (min.) was zero for all the formulations. The absence of floating lag time is ideal to prevent dosage form from the transition to the intestine which could be due to low density of prepared microspheres [14]. Obtained data of in vitro buoyancy study are given in table 1 .

Formulation F1 possesses best floating ability $(87.35 \% \mathrm{w} / \mathrm{w})$ while it gradually decreased by replacing the concentration of TI polysaccharide by eudragit. Floating ability of prepared microspheres may be co-related as per the swelling characteristic of microspheres. Due to the highest concentration of TI polysaccharide in formulation F1, these particles swell to its highest extent and become floatable. Since eudragit are not swellable therefore by increasing the concentration of eudragit, microspheres started to sediments.

\section{Encapsulation efficiency}

Data of encapsulation efficiency of prepared microspheres are shown in table 1 . Values of percentage encapsulation efficiency were increased by increasing the TI polysaccharide concentration in respect to the concentration of eudragit. The reason may be, increase in polysaccharide concentration leads to increase in the viscosity of aqueous phase and highly viscous phase shows high drug retention capacity by preventing the migration of drug from droplets to surrounding media which result to more encapsulation efficiency [12].

\section{In vitro dissolution studies}

The in vitro releases of $\mathrm{RH}$ from all batches prepared by using a mucoadhesive TI polysaccharide and a rate controlling polymer (eudragit) are illustrated in fig. 4. All the formulations showed drug released pattern till $12 \mathrm{~h}$ course of study. Initially, at $30 \mathrm{~min}$ $30.73 \% \mathrm{w} / \mathrm{w}, 24.16 \% \mathrm{w} / \mathrm{w}, 21.90 \% \mathrm{w} / \mathrm{w}$ and $17.75 \% \mathrm{w} / \mathrm{w}$ drug was released from the formulations F1, F2, F3 and F4 respectively. The rate of drug release was found gradually decreased by increasing the concentration of eudragit concentration into the prepared microspheres. Increased concentration of eudragit into microspheres make an impermeable three-dimensional network through which drug migration become difficult and as a result suppress the drug release. Since eudragit are insoluble at acidic $\mathrm{pH}$ therefore at highest concentration (F4) drug release was found to be decreased. Moreover, all prepared TI polysaccharide based formulations were found to be release $100 \% \mathrm{w} / \mathrm{w}$ of RH from 8 to 12 
$\mathrm{h}$ into acidic media. Rationally, the prepared microspheres can be delivered the drug into the upper part of GIT up to $12 \mathrm{~h}$.

The exact mechanism of drug release from prepared microspheres was calculated by fitting the release data into several models. The criterion of selection of best model was based on the goodness of fit. It was observed that drug release from microspheres followed Higuchi square root kinetics for the formulations F2, F3 and F4. While the formulation F1 (solely containing TI and not containing Eudragit) followed korsemeyer peppas kinetics having the value of $n$ was 0.352 . This shows that the release drug release kinetics of the TI microspheres (F1) was a fickian. However, the formulations F2, F3 and F4 followed diffusional kinetics according to Higuchi's equation, all the plots were linear with correlation coefficient values (r2) in the range of 0.8914-0.9452 (table 2), indicating diffusion controlled drug release. Finally, in order to know the exact mechanism of drug diffusion through prepared microspheres, the release data was fit into Korsmeyer-Peppas equation. All the plots were found to be linear with $\mathrm{r} 2$ values in the range of $0.9764-0.9936$ (table 2). The release exponent (n) values for all batches were found to be more than 0.45 , indicating Fickian release.

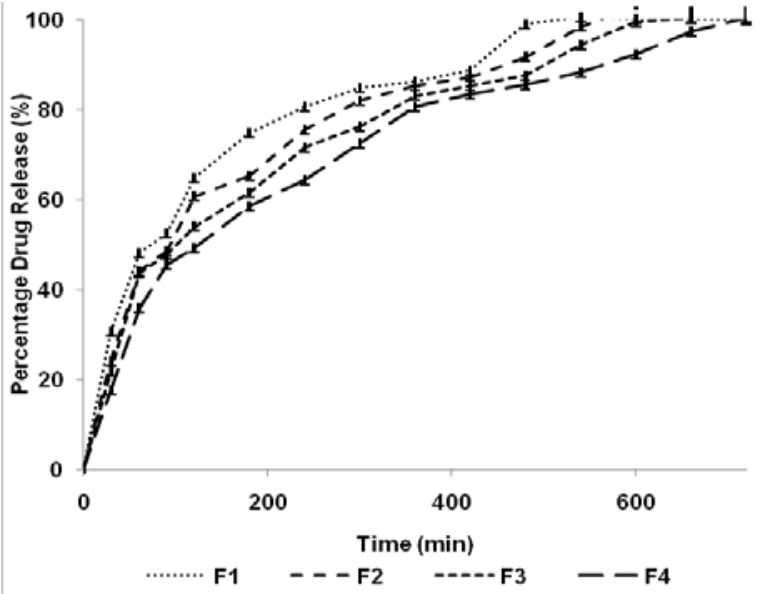

Fig. 4: Percentage release of RH from TI polysaccharide based floating-mucoadhesive microspheres $(n=3$, mean $\pm S D)$

Table 2: Values of kinetic rate constant $(k)$, correlation coefficient $\left(R^{2}\right)$ and release exponent (n) from different batches of prepared microspheres

\begin{tabular}{|c|c|c|c|c|c|c|c|c|c|}
\hline \multirow[t]{2}{*}{ Formulations } & \multicolumn{2}{|c|}{ Zero-order } & \multicolumn{2}{|c|}{ First-order } & \multicolumn{2}{|c|}{ Higuchi } & \multicolumn{3}{|c|}{ Korsmeyer-Peppas } \\
\hline & $\mathbf{K}$ & $\mathbf{R}^{2}$ & $\mathbf{k}$ & $\mathbf{R}^{2}$ & $\mathbf{k}$ & $\mathbf{R}^{2}$ & $\mathbf{K}$ & $\mathbf{R}^{2}$ & $\mathbf{n}$ \\
\hline F1 & 0.107 & 0.751 & -0.107 & 0.751 & 3.565 & 0.928 & 10.881 & 0.955 & 0.352 \\
\hline $\mathrm{F} 2$ & 0.116 & 0.819 & -0.116 & 0.819 & 3.762 & 0.963 & 2.832 & 0.961 & 0.643 \\
\hline F3 & 0.115 & 0.843 & -0.115 & 0.843 & 3.699 & 0.973 & 2.6143 & 0.923 & 0.648 \\
\hline $\mathrm{F} 4$ & 0.115 & 0.864 & -0.115 & 0.864 & 3.687 & 0.981 & 2.1149 & 0.940 & 0.659 \\
\hline
\end{tabular}

\section{CONCLUSION}

The present study concludes that the extracted plant polysaccharide of TI can be considered as excipients for the development of floating-mucoadhesive drug delivery. Extracted polysaccharide possesses an optimized value of viscosity and extraction procedure was found suitable with optimum yield. Prepared microspheres found suitable in buoyancy study with high encapsulation efficiency.

Finally, in vitro release study indicates that microspheres were able to release the RH up to $12 \mathrm{~h}$ into acidic media. Further investigations such as stability studies, scale-up studies and in vivo pharmacokinetic studies are needed for the establishment of Tamarindus indica polysaccharide as pharmaceutical excipients for microspheres drug delivery systems.

\section{CONFLICTS OF INTERESTS}

Declare none

\section{REFERENCES}

1. Maslarska V. Determination of ranitidine hydrochloride in pharmaceutical preparations by direct potentiometry. Int J Pharm Pharm Sci 2014;6:538-40.

2. Abdul WB, Larry FL. Colonic metabolism of ranitidine, the implication for its delivery and absorption. Int J Pharm 2001;227:157-65.

3. Khan K, Bajpai M. Floating drug delivery system: an overview. Int J Pharm Tech Res 2014;2:2497-505.

4. Elijah IN, Barbara RC. Evaluation of grewia polysaccharide gum as a suspending agent. Int J Pharm Pharm Sci 2011;3:168-73.

5. Malviya R, Sharma PK. Extraction and evaluation of Tamarind seed polysaccharide as a pharmaceutical in situ gel forming system. Am Eur J Sci Res 2014;9:1-5.

6. Bhargav B, Anuradha P, Narkhede S. Formulation, evaluation and optimisation of carboxy methyl tamarind powder based baclofen floating matrix tablet using $3^{2}$ full factorial designs. J Pharm Sci Biosci Res 2016;6:221-30.
7. Nayak AK, Pal DK, Santra K. Swelling and drug release behaviour of metformin HCl-loaded tamarind seed polysaccharide alginate beads. Int J Biol Macromol 2016; 82:1023-7.

8. Sharma N, Kulkarni GT, Sharma A, Bhatnagar A, Kumar N Natural mucoadhesive microspheres of Abelmoschus esculentus polysaccharide as a new carrier for nasal drug delivery. J Microencapsulation 2013;30:589-98.

9. Mazumder R, Nath LK, Haque A, Maity T, Choudhury PK, Shrestha B. Formulation and in vitro evaluation of natural polymers based microspheres for colonic drug delivery. Int J Appl Pharm 2010;2:211-9.

10. Dey SK, De PK, De A, Ojha S, De R. Mukhopadhyay AK, et al. Floating mucoadhesive alginate beads of amoxicillin trihydrate: a facile approach for H. pylori eradication. Int J Biol Macromol 2016;89:622-31.

11. Junginger HE, Verhoef JC, Thanou M. Drug delivery: mucoadhesive hydrogels. In: Swarbrick J, ed. Encyclopedia of pharmaceutical technology. New York: Informa healthcare; 2007. p. 1169-82.

12. Freitas S, Merkle HP, Gander B. Microencapsulation by solvent extraction/evaporation: reviewing the state of the art of microsphere preparation process technology. J Controlled Release 2005;102:313-8.

13. Mohamed EE, Engy HM, Sally G, Aly HN. Floating ranitidine micro particulates: development and in vitro evaluation. Int J Appl Pharm 2016;8:1-9.

14. Nasa P, Mahant S. Floating drug delivery system using Methocel K100M and E50: Formulation and characterization. Acta Pharm Suec 2011;53:57-65.

\section{How to cite this article}

- Vikram Kumar Sahu, Nitin Sharma, Pratap Kumar Sahu, Shubhini A Saraf. Formulation and evaluation of floatingmucoadhesive microspheres of novel natural polysaccharide for site specific delivery of ranitidine hydrochloride. Int J Appl Pharm 2017;9(3):15-19. 